$\xi=1$ -

\title{
Hop-based Exclusive Neighborhood Caching Scheme for Content Centric IMANET
}

\author{
Backhyun Kim ${ }^{1}$, Iksoo Kim ${ }^{1}$, Yoseop Woo ${ }^{1} *$ \\ ${ }^{1}$ Department of Information and Telecommunication Engineering, Incheon National University \\ 119 Academy-ro, Yeonsu-gu, Incheon, Korea \\ *Corresponding author E-mail: yswooo@inu.ac.kr
}

\begin{abstract}
MANETs have a relatively low transmission bandwidth and energy capacity, which is very different from a wired network. Frequent change of the transmission path due to movement of the node may cause problems such as service delay or disconnection in providing multimedia contents requiring transmission for a long time. A caching scheme that stores and uses the content being transmitted for future requests is an efficient technique in terms of use of transmission bandwidth and node energy. In this paper, caching is performed by selecting nodes that are not located within the transmission range of each other based on the hop distance among the nodes adjacent to the transmission path in the routing process. This can drastically reduce the number of neighboring nodes performing caching and decline the load on the node due to the low probability of being selected as a cache. Simulations were performed in terms of cache hit ratio, transmission channel reduction rate, and the number of caching nodes for various service request rates. From the simulation results, we can confirm that the proposed scheme adds a small number of caching nodes but substantially improves performance.
\end{abstract}

\section{Introduction}

Advances in telecommunication technologies have allowed users to use whatever services they want regardless of time and place, and have led to explosive use of multimedia data such as video and audio using streaming method. This situation inevitably requires the implementation of a high-speed communication infrastructure. To implement this, relatively long time and high cost are required. It is therefore difficult to provide services to users whenever and wherever they want. Mobile ad-hoc networks (MANETs) are a network consisting of only nodes with communication capabilities [1], [2]. MANETs are an excellent alternative to situations where communication infrastructure is difficult to implement or does not function properly such as battlefields, disaster areas, mountainous areas, etc. The node performs all operations related to the path management and transmission. This is a heavy burden for nodes using limited energy and bandwidth. Mobile nodes can be connected to a fixed network such as Internet by being wirelessly attached to an AP of wireless LAN [3]. Compared with direct access to the Internet using a communication infrastructure such as $4 \mathrm{G}$ mobile network, IEEE $802.11 \mathrm{x}$, etc., multimedia services can only be provided in a limited manner.

Transmitting the identical content whenever a request occurs causes inefficient use of node energy and network bandwidth. Many studies have been conducted to solve these problems in various network environments [4], [5], [6]. In case of multimedia contents, since the transmission duration is relatively long, the probability of a new request occurring during the item is being transmitted is larger than that of non-multimedia contents. In MANETs, the service of the requested content is provided by sequentially performing the processes of receiving and forwarding by the nodes constituting the transmission route between a server and a requester. The nodes constituting the transmission path, i.e. forwarding node, can store the items that they forward to provide for future requests. The service request is transmitted by sequentially passing through the nodes constituting the route to the server storing the contents. If one or more of the nodes constituting the path are storing the requested content, i.e. caching, the service may be provided by the forwarding node rather than being provided by the server. In this case, since the transmission is reduced by the number of paths between the server and the forwarding node, the consumption of the transmission bandwidth and the node energy are reduced by the corresponding amount [7]. The reduction of the number of transmission paths also decreases the probability of delaying or buffering the multimedia service. Even when no connection is established with the server, the requested service can be provided from the adjacent node.

In this paper, we propose a new caching scheme, which is performed in three kinds of nodes: the service request node, the forwarding nodes constituting the transmission path, and the selected nodes adjacent to the transmission path. Because the scheme adheres to content centric network, the contents being transmitted are stored among three kinds of nodes and provided as a service for a request that is generated later. As the number of nodes performing caching increases, a higher cache hit ratio can be obtained, thus increasing network utilization efficiency. If a larger number of nodes perform caching than necessary, only a relatively small number of cached contents are provided, resulting in inefficient use of node energy. Caching is performed only by nodes that are not located within the transmission range of each other among the nodes adjacent to the transmission path.

The remainder of the article is structured as follows: The next Section briefly summarize the related work of caching schemes applied to MANETs. Section 3 describes the caching scheme 
based on transmission route to reduce the burden of nodes using the finite capacity of energy. Section 4 presents the simulation results and their analysis. Finally, the paper is concluded in Section 5 .

\section{Related Work}

MANETs are an infrastructure-less self-configuring network consisting solely of mobile devices connected by wireless communication scheme. Internet-based mobile ad-hoc network (IMANET) is an Internet protocol-based network in MANETs. The network consists of two layers: mobile host and mobile router [3]. The nodes in the mobile host layer are temporarily and directly connected to the fixed network. The mobile router layer consists of mobile router nodes responsible for the connection between the node and the fixed network, and mobile hosts configuring of MANETs. Mobile hosts can be temporarily or permanently connected to a fixed network via mobile routers.

Content centric network (CCN) [7], [8], [9] is a scheme solving the disadvantage that users must know the address of the host proving the contents in order to receive the desired service. CCN provides services using the names of content irrelevant to the location. Named content is automatically populated or pre-populated in distributed caches among hosts. When a user generates a request using content name, the user is served from the nearest cache storing the content. Therefore, $\mathrm{CCN}$ can reduce transmission hops and consume less network resources such as bandwidth, processing power, energy, etc.

Cache is a collection of data replicating the original content. Caching is to create and provide these replicated data for reducing bandwidth consumption, improving performance, or using them quickly. Various caching techniques for $\mathrm{CCN}$ have been studied [10], [11], [12]. LCE (Leave Copy Everywhere) is a method in which all the nodes constituting the transmission path duplicate the content [10], [11]. In LCD (Leave Copy Down) [12], to avoid repetitive duplication of the identical content, replicas are not cached in all intermediate nodes, but stored only in the child node of the node where the cache hit occurred. MCD (Move Copy Down) [12] is similar to LCD but when cache hit occurs in k-step host, the cached content is moved to $\mathrm{k}-1$ step host, where $\mathrm{k}$ is the distance from the requesting host. This allows a redundant copy of the same content to exist at a minimum on the transmission path, and can provide a faster service due to the reduced number of hop. Due to the mobility of the nodes, it is difficult to apply the caching scheme for wired networks to MANETs. In sparse mode where a small number of nodes exist in the network, the path among the nodes cannot perpetually exist. When the nodes encounter accidently, i.e. sparse mode may not guarantee full connectivity among nodes in the network, they exchange contents that are expected to be used later based on their popularities [13]. In MANETs, content is transmitted from the server to the requesting host via intermediate nodes in a multi-hop manner. The intermediate nodes store and forward the content in this process and, therefore, can cache the content without further processing [14]. Cooperative caching techniques shares and coordinates the data being stored in mobile hosts. In order to reduce redundancy of contents and load of nodes, caching is performed by specifically selected nodes or in specific area [15], [16]. The ZoneCooperative finds the requested content only from nodes that are located within $\mathrm{n}$ hops, called zones, from the requestor [15]. This scheme isolates the caching process to a specific area without flooding the entire network. In [16], the proposed scheme allows a host with the largest number of neighboring nodes to perform caching.

The cache replacement algorithm is very important issue because the amount of storage space implemented on the host is finite. LRU (Least Recently Used) is the most widely used popular caching replacement policy due to its simplicity of implementation [7], [8], [9], [10], [11], [12], [16], [17]. Probability-based caching policies are proposed in [11], [12]. If cached items are deleted due to insufficient storage capacity, retransmission from the server is required when the removed items are requested later. The neighbor caching in [17] moves unused cache data to its neighbor node in the idle state to improve cache space utilization.

The contents provided by the server are not requested from the users at the same frequency [13]. Popularity-based Zipf's law is widely used to define different request frequencies for each content. The request rate for the contents may be variously defined using the exponential distribution, the Pareto distribution, the uniform distribution, etc.

\section{Hop-based Exclusive Neighborhood Cach- ing Scheme}

The performance of caching scheme is determined by a number of factors, and the purpose of this paper is to determine how and how many hosts should perform caching to ensure that the requested content is served by the closest host as possible. We assume that the content server is located within the wired network. In this paper, we focus on the network consisting of mobile router and mobile host, and do not consider transmission in wired network.

In MANETs, the transmission path is formed dynamically and temporally due to the movement of the nodes. Therefore, the path should be managed periodically or whenever necessary for a robust route. We consider that the path due to node movement is managed by the DSDV (Destination Sequenced Distance Vector) routing protocol [19]. We consider the situation where the content server provides $\mathrm{C}$ items. Content provided by the server has different popularity and is requested with different probabilities. We assume that a series of service requests do not temporal correlations of services requests as in the case of the Independent Reference Model (IRM). Assume that the network consists of $\mathrm{N}$ nodes, labelled $\mathrm{k}=1,2, \ldots, \mathrm{N}$. Let $\mathrm{H}$ be the set of nodes.

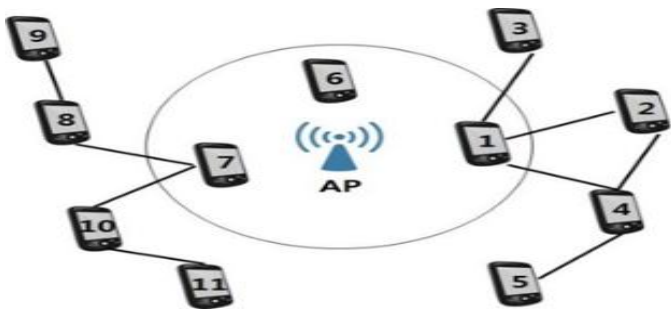

Fig. 1: An example of network topology at time $t$

Assume that the transmission radii of AP and all nodes is fixed at R. Fig. 1 shows an example of a network topology at time $t$ consisting of one AP and 11 nodes, where the solid circle represents the AP's transmission range and the solid lines represent the communication links with neighbors among the nodes. Since the nodes $H=\{1,6,7\}$ are located within the transmission range of the AP, they establish direct link with the AP to connect to the server. The remaining nodes must be connected directly or multihop to one or more of the nodes $H=\{1,6,7\}$ to be associated with the server located in the wired network as they are located outside the AP's transmission range. Assume that node $\mathrm{N}_{\mathrm{e}}$ requests an item at time $t$ when the topology between nodes is temporarily formed as shown in Fig. 1. Under the DSDV routing environment, the request packet of node 5 is sent to the parent node 4 on the path to the AP. The node 4 that receives the request packet forwards it to the parent node 1 , and this process is repeated until the AP is encountered. Content transmitted from the server is delivered in the reverse order of the previous process.

In this paper, the selected nodes can perform caching in the course of transmitting the contents called hop-based exclusive neighborhood (HEN) caching scheme. First, like LCE [12], the nodes, $H=$ $\{1,4,5\}$, constituting the transmission path cache the content, though they are not the requester. Due to the broadcast nature of the wireless transmission scheme, nodes $H=\{6,7\}$ also receive 
this content when the AP transmits a content to node 1 . Nodes directly connected to AP do not perform caching because the caching load becomes large when the request rate is high. When node 1 transmits, nodes $H=\{2,3,4\}$ receive it. Similarly, When node 4 transmits, nodes $H=\{1,2,5\}$ receive it. Caching is performed on all nodes that are not directly involved in the requested content delivery but receive the transmission data, i.e. aggressive caching. This technique can achieve a high cache hit ratio. However, as the service request rate increases, the number of nodes performing caching increases. This causes a problem of redundant storage in which the identical content is stored in a plurality of nodes. This problem can be reduced by selecting the nodes adjacent to the transmission path. To do this, we propose a caching scheme for neighboring nodes that are not located within the transmission range of each other based on the hop count.

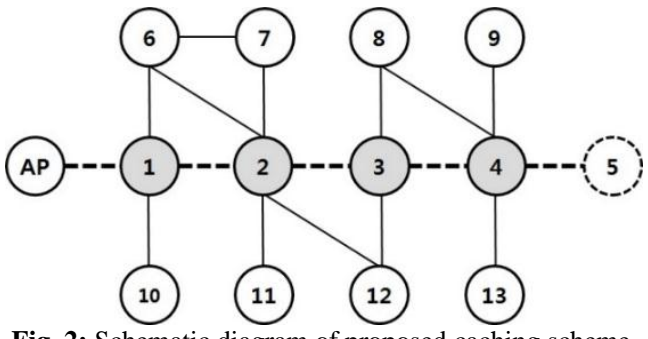

Fig. 2: Schematic diagram of proposed caching scheme

Fig. 2 shows a schematic diagram of proposed caching scheme with 4 forwarding nodes and 8 neighboring nodes where the requester is node 5. From the Fig. 2, the requester is connected to the AP through links that are sequentially connected between nodes $4,3,2$ and 1 . The gray circle represents the forwarding node constituting a transmission path, and the broken circle represents the requester. The thick dashed lines represent the links from the server to the requester, and the solid lines indicate the links of neighboring nodes. The movement of the node causes the distance from the fixed AP to approach or move away. Therefore, it is inefficient for the neighboring nodes of forwarding nodes located in each hop to perform caching. $\varphi$ represents the location, i.e. hop, to perform caching. Let $\varphi$ be 1 . From Fig. 2, neighboring nodes $H=$ $\{6,10\}$ of node 1 are selected as nodes performing caching. In the second hop, node $H=\{11,12\}$ is selected. Node 7 is not selected as a caching node because node 6 has already been selected as the caching node in Fig. 2. Subsequently, node $H=\{8\}$ is selected in the third hop and nodes $H=\{9,13\}$ is selected in the fourth hop. If $\varphi$ is 2 , the caching node selection process is performed every second hop. In the second hop, nodes $H=\{6,11,12\}$ or $H=\{7$, $11,12\}$ is selected in Fig. 2. Nodes $H=\{8,13\}$ or $H=\{9,13\}$ is selected in the fourth hop. Where $\varphi$ is 3 , nodes $H=\{8,12\}$ is selected. Where $\varphi$ is 4 , nodes $H=\{8,13\}$ or $H=\{9,13\}$ is selected. The number of caching nodes is $7,5,2$, and 2 , respectively, depending on the value of $\varphi$. In Fig. 2, if an aggressive technique is used to allow all neighboring nodes to perform caching, eight neighboring nodes perform it. The proposed scheme can reduce the number of caching nodes by 1, 3, 6 and 6 in each $\varphi$ compared with the aggressive technique.

$P_{t}=P_{p}+P_{n}=\sum_{i=1}^{H-1}\left(1-\bar{p}_{c}\right)^{i} \bar{p}_{c}+\sum_{j=1}^{\left\lfloor\frac{H}{\varphi}\right\rfloor} \sum_{i=1}^{\bar{m}}\left(1-\bar{p}_{c}\right)^{i} \bar{p}_{c}$

Let $\mathrm{H}$ and $\bar{P} c$ be the number of hops from server to the requester, and the mean caching probability, respectively. The probability $P_{t}$ that the requested content will be cache hit under the proposed scheme is shown in Eq. 1. Let $\bar{m}$ be the mean number of neighboring cache node in each hop, and $P_{p}$ and $P_{n}$ be the probability of cache hit in the transmission path and neighboring node, respectively.

Assume that the transmission path is maintained for an average of $\mathrm{t}$ times and the content is transmitted at the constant bit rate of $R$ bits/sec. The consumed network bandwidth can be calculated as

Eq. 2, where $h$ is the number of hops from a cache hit node to the requester, and $\mathrm{Q}_{\mathrm{nc}}$ and $\mathrm{Q}_{\mathrm{c}}$ are the amount of transmission bandwidth consumed when a cache miss occurs and when a cache hit occurs, respectively.

$$
Q_{n c}-Q_{c}=(H-h) R t
$$

\section{Simulation and Analysis}

In this section, we show simulation results of demonstrating the benefit of proposed hop-based exclusive neighborhood caching scheme for Internet-based mobile ad-hoc networks and analyze the results of performance using it. Simulation parameters are listed in Table 1 . We set the values of some of these variables variously to perform sensitivity analysis. The size of simulation network is $100 \mathrm{~m} \times 100 \mathrm{~m}$ in rectangular plan. The routing process is periodically performed every 1 second in DSDV routing environment [19]. Initially, the mobile nodes are randomly distributed in the network according to the Poisson distribution. AP connecting MANETs and Internet is selectively located in $(50,50)$ and $(25$, 50) to analyze the characteristics according to the node's movement. The access pattern of the multimedia contents follows cutoff Zipf-like distribution with a skew factor of 0.8 [13]. We defined cache write policy and node mobility using LRU and RWP, respectively. We take the average of 10 simulations performed for 1000 seconds.

Table 1: Simulation parameters

\begin{tabular}{|c|c|c|c|c|}
\hline Parameter & Symbol & Default & Range & Unit \\
\hline Number of nodes & $\mathrm{N}$ & 250 & $200 \sim 300$ & - \\
\hline Number of contents & $\mathrm{C}$ & 100 & $100 \sim 150$ & - \\
\hline Number of frame & $\mathrm{F}$ & 30 & fixed & frames/second \\
\hline Capacity of cache & $\mathrm{CC}$ & 300 & $200 \sim 400$ & frame \\
\hline Transmission range & $\mathrm{R}$ & 15 & $10 \sim 20$ & meter \\
\hline Request rate & $\lambda$ & 6 & $6 \sim 12$ & requests/minute \\
\hline Velocity & $\mathrm{V}$ & 4 & $0 \sim 8$ & meter/second \\
\hline Interval of caching & $\varphi$ & 2 & $1 \sim 3$ & hop counts \\
\hline
\end{tabular}

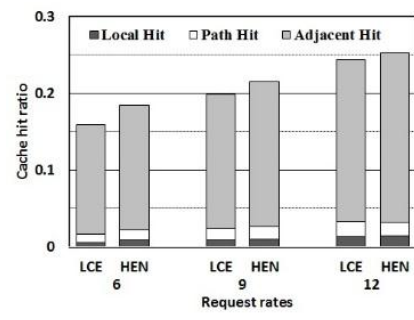

(a) The cache hit ratios expressed in local hit, path hit, and adjacent hit for various request rates

Fig. 3: Cache hit ratio

Fig. 3(a) shows the total cache hit ratios computed by the LCE and the proposed caching method HEN for local, path, and adjacent hits when the service request rates $\lambda$ are $\{6,9,12\}$. Unlike a wired network, nodes constituting a transmission path are not fixed because they are frequently changed due to the free movement. In the simulation, the cache capacity of the node CC is $10 \%$ compared with the number of contents $\mathrm{C}$ provided by the server. The use of caches shows that they can provide better performance compared to the implemented cache capacity at various request rates. From Fig. 3(a), as the request rate increases, the performance difference between HEN and LCE decreases. As the request rate increases, the difference between the caching schemes becomes smaller due to the increase in the number of nodes composing the transmission path. The simulation results in Fig. 3(a) show that the proposed caching technique has relatively better performance when the request rate is low.

Fig. 3(b) shows the cache hit ratio according to the location of the $\mathrm{AP}$ when the nodes performing caching process are selected by the 
number of transmission hops where the request rate is 6 . The proposed scheme is more affected by the location of the AP than the interval of the nodes, $\varphi$, performing the caching process. RWP has a relatively high probability of being located near the center of the network. In this case, as shown in Fig. 3(b), a large number of requests are provided from the AP, not from the caching node, thus resulting in a low cache hit ratio.

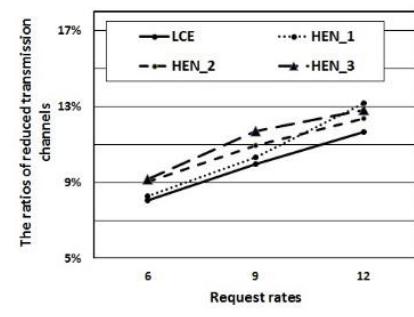

(a) Reduced transmission channel ratio by caching scheme

Fig. 4: Simulation results according to various request rates

Fig. 4(a) shows the reduction ratio in the number of transmission channels when caching schemes are used. HEN_1, HEN_2, and HEN_3 represent the hop distance $\varphi$ selected as a caching node in the proposed scheme, respectively. In all cases as shown in Fig. 4(a), the reduction rate of the transmission channel and the service request rate are proportional to each other. Fig. 4(b) shows the probability of being selected as a caching node according to the hop interval $\varphi$ during the simulation. Experimental results in Fig. 4(b) show that there is no significant difference between hop intervals 1 and 2 because the proposed caching scheme cannot select different caching nodes within the transmission range of the caching node. Simulation results show that the proposed scheme has advantages in terms of the cache hit rate and the number of transmission channels compared to the number of caching nodes to which the proposed scheme is added.

\section{Conclusion}

In this paper, we designed the hop-based exclusive neighborhood caching scheme. The caching nodes are designated among the neighboring nodes of the forwarding node that are not located within the transmission range of each. In addition, the hop count based selection scheme decreases the number of nodes performing caching, thereby reducing the load on the node. Simulation results show that the proposed scheme has better performance where the request rate is lower and the transmission paths centering on the $\mathrm{AP}$ are asymmetric.

\section{Acknowledgement}

This work was supported by Incheon National University Research Grant in 2017

\section{References}

[1] Ghosekar, Pravin, G. Katkar, and P. Ghorpade, "Mobile ad hoc networking: imperatives and challenges", IJCA Special issue on MANETs, Vol.3, (2010), pp.153-158.

[2] M. Conti and S. Giordano, "Mobile ad hoc networking: milestones, challenges, and new research directions", IEEE Communications Magazine, Vol.52, No.1, (2014), pp.85-96.

[3] M. S. Corson, J. P. Macker, and G. H. Cirincione, "Internet-based mobile ad hoc networking", IEEE internet computing, Vol.3, No.4, (1999). pp.63-70.

[4] G. Adam, C. Bouras, A. Gkamas, V. Kapoulas, G. Kioumourtzis and N. Tavoularis, "Performance evaluation of routing protocols for multimedia transmission over mobile ad hoc networks", In Wireless and Mobile Networking Conference (WMNC), 2011 4th Joint IFIP, IEEE, (2011), pp.1-6.
[5] L. Shrivastava, G. S. Tomar, and S. S. Bhadoria, "A load-balancing approach for congestion adaptivity in MANET", In Computational Intelligence and Communication Networks (CICN), (2011), pp.3236.

[6] M. Rath and B. K. Pattanayak, "A methodical survey on real time applications in MANETS: Focussing on key issues", In High Performance Computing and Applications (ICHPCA), (2014), pp.1-5.

[7] C. Fricker, P. Robert, J. Roberts, and N. Sbihi, "Impact of traffic mix on caching performance in a content-centric network", In Computer Communications Workshops (INFOCOM WKSHPS), (2012), pp.310-315.

[8] G. Carofiglio, M. Gallo, L. Muscariello, and D. Perino, "Modeling data transfer in content-centric networking", In Proceedings of the 23rd international teletraffic congress, (2011), pp.111-118.

[9] S. Y. Oh, D. Lau, and M. Gerla, "Content centric networking in tactical and emergency manets", In Wireless Days (WD), 2010 IFIP, IEEE, (201), pp.1-5.

[10] V. Jacobson, D. K. Smetters, J. D. Thornton, M. F. Plass, N. H. Briggs, and R. L. Braynard, "Networking named content", In Proceedings of the 5th international conference on Emerging networking experiments and technologies, ACM, (2009), pp.1-12.

[11] V. Martina, M. Garetto, and E. Leonardi, "A unified approach to the performance analysis of caching systems", In INFOCOM, 2014 Proceedings IEEE, (2014), pp.2040-2048.

[12] N. Laoutaris, H. Che, and I. Stavrakakis, "The LCD interconnection of LRU caches and its analysis", Performance Evaluation, Vol.63, No.7, (2006), pp.609-634.

[13] Y. Ma and A. Jamalipour, "A cooperative cache-based content delivery framework for intermittently connected mobile ad hoc networks", IEEE Transactions on Wireless Communications, Vol.9, No.1, (2010), pp.366-373.

[14] Y. C. Hu and D. B. Johnson, "Caching strategies in on-demand routing protocols for wireless ad hoc networks", In Proceedings of the 6th annual international conference on Mobile computing and networking, (2000), pp.231-242.

[15] S. Lim, W. C. Lee, G. Cao, and C. R. Das, "Performance comparison of cache invalidation strategies for internet-based mobile ad hoc networks", IEEE international conference on Mobile Ad-hoc and Sensor Systems (MASS), (2004), pp.104-113.

[16] L. Zhou, T, Zhang, X, Xu, Z. Zeng, and Y. Liu, "Broadcasting based neighborhood cooperative caching for content centric ad hoc networks", In Communications in China (ICCC), 2015 IEEE/CIC International Conference on. IEEE, (2015), pp.1-5.

[17] J. Cho, S. Oh, J. Kim, H. H. Lee, and J. Lee, "Neighbor caching in multi-hop wireless ad hoc networks", IEEE Communications Letters, Vol.7, No.11, (2003), pp.525-527.

[18] S. Vanichpun and A. M. Makowski, "The output of a cache under the independent reference model: where did the locality of reference go?", In ACM SIGMETRICS Performance Evaluation Review, Vol.32, No.1, (2004), pp.295-306.

[19] S. Gupta, B. S. Dhaliwal, and R. Malhotra, "Performance Comparison of Proactive Routing Protocols: OLSR, DSDV, WRP", International Journal of Advanced Research in Computer Science, Vol.6, No.8, Nov-Dec, (2015), pp.73-77. 